Bryn Mawr College

Scholarship, Research, and Creative Work at Bryn Mawr

College

Physics Faculty Research and Scholarship

Physics

2008

\title{
The relationship between crystal structure and NMR relaxation in molecular solids with tert-butyl groups
}

Arnold L. Rheingold

Antonio G. DiPasquale

Peter A. Beckmann

Bryn Mawr College, pbeckman@brynmawr.edu

Let us know how access to this document benefits you.

Follow this and additional works at: http://repository.brynmawr.edu/physics_pubs

Part of the Physics Commons

\section{Custom Citation}

Arnold L. Rheingold, Antonio G. DiPasquale, and Peter A. Beckmann. 2008. "The relationship between crystal structure and NMR relaxation in molecular solids with tert-butyl groups." Chemical Physics 345.1: 116-118.

This paper is posted at Scholarship, Research, and Creative Work at Bryn Mawr College. http://repository.brynmawr.edu/physics_pubs/103

For more information, please contact repository@brynmawr.edu. 


\title{
The Relationship Between Crystal Structure and NMR Relaxation in Molecular Solids with tert-Butyl Groups
}

http://www.sciencedirect.com/science/article/pii/S0301010408000827

Arnold L. Rheingold and Antonio G. DiPasquale

Department of Chemistry and Biochemistry, University of California, San Diego, 9500 Gilman Drive, La Jolla, CA 92093-0358, USA.

\author{
Peter A. Beckmann \\ Department of Physics, Bryn Mawr College, 101 North Merion Ave, \\ Bryn Mawr, PA 19010-2899, USA.
}

\section{Abstract}

We correlate an X-ray determination of the molecular and crystal structures of 2tert-butylanthracene and 2-tert-butylanthraquinone reported here with the previously reported dynamical nuclear magnetic resonance determination of the motions of the tertbutyl groups and their resident methyl groups in the solid state [P. A. Beckmann, K. S. Burbank, M. M. W. Lau, J. N. Ree, T. L. Weber, Chem. Phys. 290 (2003) 241].

\section{Introduction}

Solid state nuclear magnetic resonance (NMR) ${ }^{1} \mathrm{H}$ spin-lattice relaxation experiments determine parameters related to the rotational dynamics of tert-butyl groups and their constituent methyl groups [1 - 3]. X-ray crystallography determines the time averaged positions of atoms in molecular solids which can be used to place constraints on the dynamical models in cases where more than one model fits the NMR relaxation data [3]. In turn, unusual NMR relaxation rate data can provide the incentive for a careful X-ray analysis to determine an unusual structure [4]. Recently, a ${ }^{1} \mathrm{H}$ spin-lattice relaxation study 
of solid 2-tert-butylanthracene (2TA) and 2-tert-butylanthraquinone (2TAQ) appeared [1] at a time when we were unable to grow crystals of sufficient size to complete a highresolution, single-crystal structural determination. Since that time we have discovered new solvent combinations for recrystallizations and we have now determined the molecular and crystal structure of these two van der Waals molecular solids. As a consequence, we can confirm the surprisingly simple dynamical model for 2TAQ and we can determine the appropriate dynamical model for 2TA from among the several possibilities [1].

\section{Crystallography}

Crystallographic data has been deposited with the Cambridge Crystallographic Data Centre (see Section 5). Both compounds crystallize in the monoclinic space group $P 2_{1} / c$. 2TAQ crystallizes with two crystallographically independent molecules in the asymmetric unit and 2TA with three. 2TAQ forms a two-fold rotational twin. The crystal structure of 2TAQ is shown in Fig. 1 and the two independent molecules are shown in Fig.

2. The crystal structure of 2TA is shown in Fig. 3 where the three independent molecules can be identified. Crystals were grown from toluene/ethanol solutions into which heptane was slowly diffused under controlled temperature conditions over a period of many days. Data were collected on a Bruker Kappa APEXII diffractometer equipped with a coated capillary collimator using Cu radiation at $100 \mathrm{~K}$. Data for 2TAQ were integrated using TWINABS after determination by CELL_NOW that all samples tested were $180^{\circ}$ rotationally twinned. 


\section{Dynamics in 2TA and 2TAQ and the Relation to the Crystal Structure}

The observed temperature and nuclear magnetic resonance (NMR) frequency dependence of the ${ }^{1} \mathrm{H}$ spin-lattice relaxation rate for both 2TAQ and 2TA has been reported [1]. We denote the NMR activation energies for rotation by $E_{i}^{j}$. The superscript $j$ labels the molecule in the crystal. There are two inequivalent molecules in the unit cell for 2TAQ $(j=A, B)$ (Fig. 2) and three for 2TA $(j=A, B, C)$ (Fig. 3). The subscript $i$ refers to the four rotors in a tert-butyl group. The activation energy for tert-butyl group rotation is characterized by the subscript $i=t$ and the activation energy for the rotation of the three methyl groups is characterized by the subscripts $i=1,2,3$.

For both $2 \mathrm{TA}$ and $2 \mathrm{TAQ}$, the aromatic ring systems are nearly planar and the tertbutyl groups are oriented with one methyl group nearly in the aromatic plane and the other two methyl groups well out of this plane. The tert-butyl group $(t)$ and the in plane methyl group (methyl group 1) are characterized by an activation energy $E_{t}^{j}=E_{1}^{j}$ and the two out-of plane methyl groups ( 2 and 3 ) are characterized by the activation energy $E_{2}^{j}=$ $E_{3}^{j}$. The crystal structure of 2TAQ (Fig. 1) shows eight molecules per unit cell with two inequivalent molecules A and B. However, the tert-butyl groups of both molecules have very similar environments. As such, it is reasonable that only a single site model as previously proposed [1], fits the NMR relaxation rate data. In this case $E_{t}^{1}=E_{1}^{1}=E_{2}^{1}=E_{3}^{1}$ $=16 \mathrm{~kJ} /$ mole [1] and this is a text-book example of the correlated or geared motion of a $t$ butyl group and its three methyl groups.

The dynamical NMR model for 2TA is much more complicated and the temperature and NMR frequency dependence of the ${ }^{1} \mathrm{H}$ spin-lattice relaxation rate was previously fitted to several models involving different numbers of sites $j$ and different numbers of motions $i[1]$. Indeed, the exercise was to show that without further knowledge of the structure, the NMR relaxation experiments could not distinguish between the various 
models [1]. The structure (Fig. 3) shows three inequivalent molecules in a unit cell which contains 12 molecules. This is a somewhat unusual crystal structure and is consistent with the complex temperature dependence of the ${ }^{1} \mathrm{H}$ relaxation rate. The most reasonable dynamical model consistent with the NMR relaxation results [1] is that for each of the three sites, the in-plane methyl groups and the tert-butyl group are characterized by one activation energy $E_{t}^{j}=E_{1}^{j}$ and the out-of-plane methyl groups are characterized by a different activation energy $E_{2}^{j}=E_{3}^{j}$ for $j=A, B, C$. An odd number of sites was never considered [1] but in light of the new X-ray data, we can now say that one-third of the molecules have activation energies of about $E_{t}^{A}=E_{1}^{A}=25 \mathrm{~kJ} / \mathrm{mol}$ for their tert-butyl groups and in-plane methyl groups and activation energies of about $E_{2}^{A}=E_{3}^{A}=11$ $\mathrm{kJ} / \mathrm{mol}$ for their out-of-plane methyl groups. Two-thirds of the molecules have activation energies of about $E_{t}^{B, C}=E_{1}^{B, C}=21 \mathrm{~kJ} / \mathrm{mol}$ for their tert-butyl groups and their in-plane methyl groups and activation energies of about $E_{2}^{B, C}=E_{3}^{B, C}=10 \mathrm{~kJ} / \mathrm{mol}$ for their out-ofplane methyl groups. The actual fit is indistinguishable from that presented in Figs. 4 and 5 of reference 1 . Given that the tert-butyl groups on $\mathrm{B}$ and $\mathrm{C}$ molecules are close neighbors and are in similar environments (Fig. 3) and that the tert-butyl groups on A molecules appear to be in quite different sites than the tert-butyl groups on $B$ and $C$ molecules, it is reasonable to associate the parameters for one-third of the molecules $E_{i}^{A}$ to the $A$ sites and the parameters for two-thirds of the molecules $E_{i}^{B, C}$ to $\mathrm{B} / \mathrm{C}$ sites.

\section{Summary}

The molecular and crystal structures of 2-tert-butylanthracene (2TA) and 2-tertbutylanthraquinone (2TAQ) have been determined by X-ray diffraction. The temperature dependence of the NMR ${ }^{1} \mathrm{H}$ spin-lattice relaxation rates at two NMR frequencies have been previously reported for both solids [1]. The two studies can be combined to justify 
the model used to interpret the NMR relaxation data in 2TAQ [1]. The previous NMR study proposed several possible dynamical models for the tert-butyl groups in 2TA and made the point that dynamical NMR could not distinguish between them [1]. The crystal structure reported here shows three inequivalent molecules and we are able to find a successful model whereby, for two of the three sites, the tert-butyl group and the in-plane methyl groups have one activation energy and the two out-of-plane methyl groups have a lower activation energy. For the third site, the molecules have a different activation energy pair.

Current and future work involves performing electronic structure calculations to calculate activation energies for tert-butyl group and methyl group rotation. This requires including non-bonded intermolecular interactions in clusters of molecules. Properly incorporating intermolecular Van der Waals interactions with the number of atoms needed is particularly challenging.

\section{Supplementary Material}

CCDC 675773 (2TA) and 675772 (2TAQ) contain the supplementary crystallographic data. These data can be obtained free of charge via www.ccdc.cam.ac.uk/data_request/cif, or by emailing data_request@ccdc.cam.ac.uk, or by contacting The Cambridge Crystallographic Data Centre, 12, Union Road, Cambridge CB2 1EZ, UK; fax: +44 1223336033. 


\section{References}

[1] P. A. Beckmann, K. S. Burbank, M. M. W. Lau, J. N. Ree, T. L. Weber, Chem. Phys. 290 (2003) 241.

[2] P. A. Beckmann, C. A. Buser, K. Gullifer, F. B. Mallory, C. W. Mallory, G. M. Rossi, A. L. Rheingold, J. Chem. Phys. 118 (2003) 11129.

[3] P. A. Beckmann, C. Paty, E. Allocco, M. Herd, C. Kuranz, A. L. Rheingold, J. Chem. Phys. 120 (2004) 5309.

[4] A. L. Rheingold, J. S. Figueroa, C. Dybowski, P. A. Beckmann, Chem. Comm. (2000) 651. 


\section{Figures}

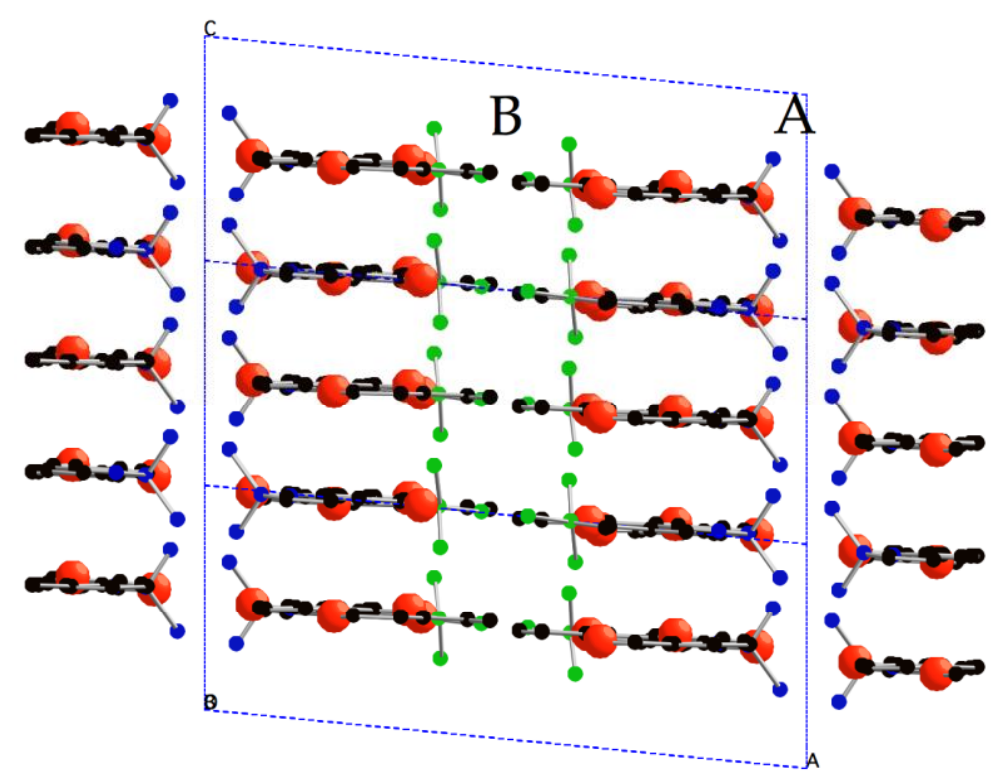

Fig. 1. (Colour online) The 010 plane of 2-tert-butylanthraquinone (2TAQ) showing 1.6 unit cells in the $x$-direction (horizontal) and 3.0 unit cells in the $z$-direction (vertical). The tert-butyl group carbon atoms of A and B molecules are labeled and shown in blue and green respectively. All aromatic carbon atoms are in black and all oxygen atoms are in red. Hydrogen atoms are omitted for clarity. Molecular fragments have been removed in the $z$-direction but not in the $x$-direction. 


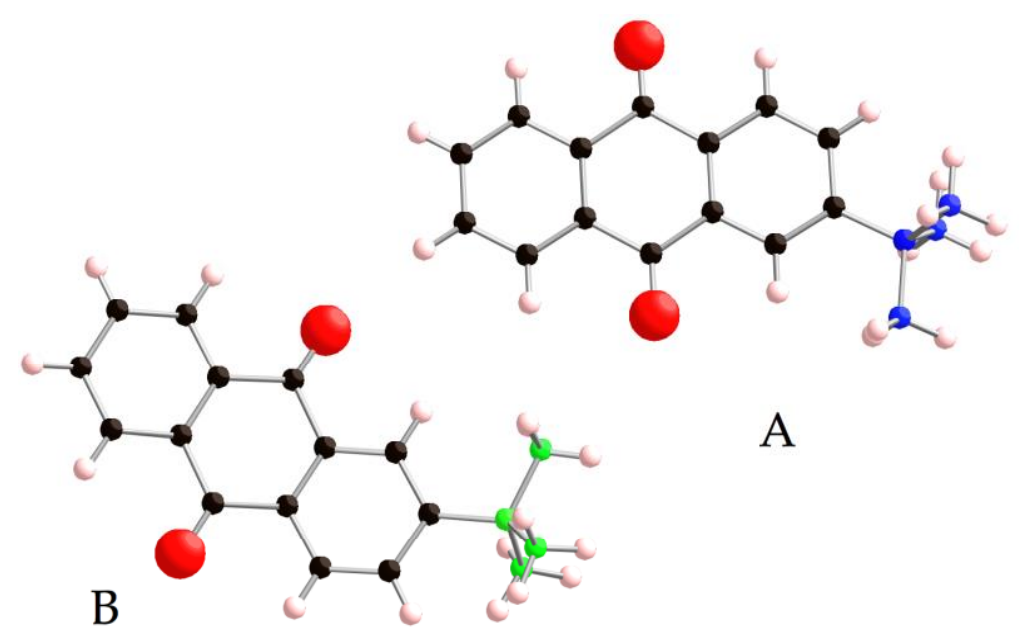

Fig. 2. (Colour online) A view looking in a direction perpendicular to the aromatic plane of the two inequivalent molecules $A$ and $B$ of 2-tert-butylanthraquinone (2TAQ) as they appear in the crystal. The tert-butyl group carbon atoms of $A$ and $B$ molecules are shown in blue and green respectively. All aromatic carbon atoms are in black, all oxygen atoms are in red, and all hydrogen atoms are in light pink. 


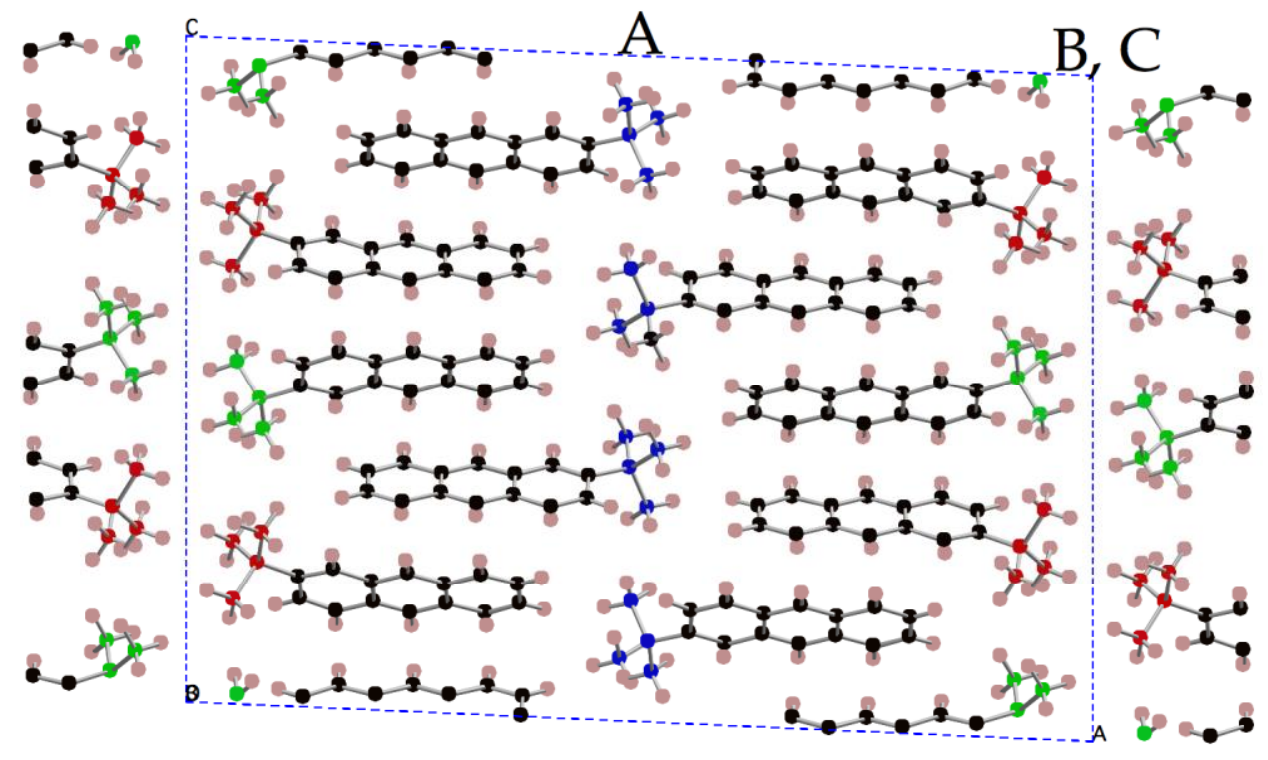

Fig. 3. (Color online) The 010 plane of 2-tert-butylanthracene (2TA) showing 1.4 unit cells in the $x$-direction (horizontal) and 1.0 unit cells in the $z$-direction (vertical). The tert-butyl group carbon atoms of $A, B$, and C molecules are labeled and shown in blue, red, and green respectively. All aromatic carbon atoms are in black and hydrogen atoms are in light pink. Molecular fragments have not been removed. 